etc.), la faculté que sa crème a de monter est moins réduite que par toute autre méthode de pasteurisation basse, ce qui, à son tour, le rend plus apte à subir l'épreuve de ma réaction crémométrique. pour la pasteurisation lente.

$4^{0}$ Toute infection après la pasteurisation du lait est éliminée.

$5^{\circ}$ Le fait que la bouteille est également pasteurisée augmente le pouvoir de conservation du lait.

\title{
DES CRITÉRIUIVS DONT ONTDEVRAIT S'INSPIRER ET DES MÉTHODES QUE L'ON DEVRAIT SUIVRE DANS L'APPRÉCIATION D'UN PROCÉDÉ DE PASTEURISATION DU LAIT
}

\author{
par HENRI STASSANO.
}

En écrivant cet article, je m'adresse à tous ceux qui, par leurs études ou leurs fonctions, sont appelés à se prononcer sur la valeur de tel ou tel procédé, de tel ou tel appareil de pasteurisation du lait, au double point de vue de l'hygiène et de la pratique industrielle, lesquelles, désormais, sont inséparables. Il est, à mon avis, d'une urgente nécessité que les savants et,les spécialistes du lait se mettent d'accord sur le choix des critériums, à la fois scientifiques et pratiques, qui seuls peuvent permettre un jugement sûr, une appréciation valable, impartiale. J'exprime le souhait que le prochain Congrès international de Laiterie mette ce sujet à l'ordre du jour de ses discussions. Déjà, au Congrès de 1931, à Copenhague, soit en séance publique, soit dans des conversations particulières, j'ai appelé l'attention sur la nécessité qu'il y a, d'ores et déjà, d'envisager : $1^{\circ}$ la conservation de l'acide carbonique ; $2^{\circ}$ l'absence concomitante d'aération dans un lait convenablement pasteurisé.

Satisfaire à ces deux observations qui, jusqu'ici, n'ont, pour ainsi dire, jamais été faites, e'est assurer à une pasteurisation du lait réalisée rationnellement deux caractéristiques importantes, mais qui ne sont pas seules, ainsi que nous allons le voir par la suite. Quelques explications feront mieux connaître l'importance de ce que j'avance, sans crainte d'être exposé, lecture faite, au reproche d'exagération ou de partialité.

A. Par la conservation aussi parfaite que possible de l'acide carbonique pendant une pasteurisation bien faite du lait, d'une part, les sels de chaux et les phosphates se maintiennent dans le lait dans leur état antérieur; d'autre part, les équilibres chimiques du lait étant, dans une certaine mesure, sous la dépendance de l'acide carbonique de cette liqueur, si ce gaz n'abandonne point ses labiles combinaisons, le lait conserve toute sa valeur biologique, ainsi que 
d'autres éléments qu'il renferme et que l'on considère soit comme des hormones, soit comme des principes bactéricides ou immunisateurs. L'expérience de A. Lumrìne qui montre qu'un sérum doué d'un grand pouvoir anaphylactique, le perd au point de devenir inoffensif s'il est soumis à l'action du vide, - laquelle, vraisemblablement, ne peut que priver ce sérum de son acide carbonique, - doit rendre bien attentif les hygiénistes sur la répercussion fâcheuse que peut avoir sur la conservation dans le lait des principes si délicats rappelés ci-dessus, le départ plus ou moins complet de l'acide carbonique. La présence de ces principes, d'une si grande utilité pour favoriser ou protéger la croissance des jeunes êtres, a été ignorée jusque dans ces derniers temps, - et elle l'est encore malheureusement trop, - de ceux qui s'occupent du lait, non seulement au point de vue industriel, mais aussi au point de vue hygiénique. Il ne faut sans doute pas trop s'en étonner, puisque nous voyons combien peu ont le souci, dans le monde de la laiterie, de la chute de l'acidité.

Dans tout lait chauffé à l'air libre ou par les procédés usuels de la pasteurisation, l'acidité tombe d'une façon sensible; cette chute ne se produit cependant pas, ainsi que je l'ai montré, même si l'on porte le lait à des températures élevées dans le but de le stériliser, lorsque son chauffage est effectué d'après les règles strictes du procédé de la couche mince que j'ai étudié. M. FouAssrer a eu l'occasion, dans un examen qu'il pratiqua en 1921 à mon laboratoire, de constater, - avec étonnement, me permettrai-je de dire, - le maintien de l'acidité initiale dans un lait néanmoins chauffé à $140^{\circ}$, en couche mince il est vrai. Mais il suffit de s'éloigner quelque peu des caractéristiques essentielles du procédé de la couche mince pour que la chute de l'acidité du lait se produise également, comme dans les pasteurisateurs usuels. En effet, pour que l'on obtienne cette conservation de l'acide carbonique, - à laquelle, sans exagération, je crois que l'on doive attacher de l'importance, - il ne suffit pas, comme cela est réalisé dans certains procédés qui tendent à se rapprocher de celui de la couche mince, d'opérer simplement le chauffage du lait en circuit fermé ; il faut qu'une telle circulation soit réalisée aux strictes conditions suivantes :

\section{a) Couche mince du lait, ne dépassant pas un millimètre d'épaisseur ;}

b) Chauffage de la couche, de part et d'autre, sur ses deux faces ;

c) Absence de poches dans le circuit, au passage de la nappe annulaire, d'un cheminement tubulaire dans un autre ;

d) Pression du lait pendant le chauffage, dépassant $1 \mathrm{~kg}$. 
- de préférence 2 à 3 - avec refroidissement immédiat dans un appareil réfrigérant sous la même pression et sous une épaisseur de lait, toujours en couche annulaire, mais pouvant, cette fois, atteindre 2 à $3 \mathrm{~m} / \mathrm{m}$ au plus.

Les communs échangeurs de température, tels les condenseurs à contre-courant de l'industrie du froid, plus ou moins modifiés, - emprunteraient-ils même certains détails à mes propres appareils, - ne remplissent nullement les conditions voulues pour éviter la séparation plus ou moins complète de l'acide carbonique, pendant la pasteurisation. Aussi, le lait chauffé dans ces conditions accuse également une certaine chute de son acidité.

Du reste, dans mon mémoire de 1926 (1), j'avais mis le lecteur en garde contre l'apparente ressemblance qu'il y a entre mes propres dispositifs et le genre commun des réchauffeurs ou échangeurs de température du type à tubes concentriques.

B. Par l'absence, d'autre part, d'aération dans le lait pasteurisé, - absence qui caractérise aussi bien que la conservation intégrale, ou presque, de l'acide carbonique, le traitement thermique du lait en vraie couche mince, - on épargne aux vitamines, - dont nul n'ignore aujourd'hui la présence dans le lait, non plus que leur grande utilité au point de vue diététique, - la plus dure épreuve qu'elles puissent subir du fait, justement, du chauffage. Les vitamines sont en général très sensibles à l'action de l'oxygène de l'air, sensibilité qu'exagère l'élévation de température.

Je faisais remarquer au Congrès de Copenhague, - remarque que j'avais du reste déjà faite dans cette même ville quelques mois auparavant à l'oceasion de la conférence que j'eus l'honneur de faire sur le lait, à l'Institut d'Hygiène de l'Université, - que si les viniculteurs, eux, se soucient fort de cette fâcheuse action combinée du chauffage et de l'aération, et s'ils font tout leur possible pour l'éviter, c'est à cause de la très forte répercussion qu'elle aurait sur la qualité du produit traité. Dans les vins, elle se traduit par un goût peu agréable de résiné, lequel goût affecte particulièrement les vins les plus fins. Quand il s'agit du lait, où joue cette même fâcheuse action combinée du chauffage et de l'aération, personne n'en a cure : les constructeurs d'appareils de pasteurisation, non plus que ceux qui s'en servent, car ni les uns ni les autres n'ont pensé suffisamment à certains critériums auxquels doivent répondre les appareils. Et cependant, il y a déjà plusieurs années, OsBorne et L. B. Mendel, les deux savants américains à qui nous devons beaucoup de connaissances précises et précieuses sur les vitamines, n'ont pas manqué de signaler aux hygiénistes comme un danger

(1) Le Lait. 1926, 6, 350, 533, 608 et 861 . 
pour la santé publique, le très grand appauvrissement que le lait subit dans son facteur antiscorbutique, pendant la pasteurisation usuelle, "où le lait est chauffé dans des conditions telles qu'il y a oxydation ". Il est même surprenant que dans les recherches faites à l'Institut de Laiterie de Kiel, si parfaitement agencé, l'attention n'ait pas été attirée sur ce côté (1).

$$
*^{*} *
$$

\section{LES CRITERIUMS QU'IL FAUT CHOISIR ET LES METHODES}

\section{A SUIVRE.}

Je"dirai tout d'abord que l'étude d'un procédé de"pasteurisation doit être conduite en suivant les règles strictes de la méthode expérimentale. C'est de cela que je me suis inspiré dans l'étude du procédé de la couche mince. Dans mon mémoire de 1926 relatif à cette question, j'ai comparé de la façon la plus étroite les résultats donnés par le procédé de la couche mince avec ceux obtenus simultanément avec le même lait par les deux procédés jusque là usuels de la pasteurisation : la pasteurisation haute et la pasteurisation basse. A la Laiterie centrale de Strasbourg, où j'ai fait mes premiers essais et que je ne saurais trop remercier, en la personne de son Directeur, M. HeIntz, pour l'obligeance et le soin apportés à faciliter mes études, ces deux procédés de pasteurisation étaient utilisés. Bien entendu, toutes comparaisons étaient faites également avee le lait cru originel qui devait donc être pasteurisé à haute température, à basse température et par le procédé de la couche mince. Cependant, la mise en pratique de la méthode comparative, dont les résultats sont de si grande valeur, suppose avant tout que les différents échantillons de lait à soumettre aux différentes épreuves sont prélevés et conservés aseptiquement. Il importe que la propre flore des laits se maintienne telle qu'elle est jusqu'aux essais, et qu'aucune contamination n'intervienne lors des prélèvements. La prise d'échantillons des laits dans des tubes doit donc être faite avec toutes les précautions nécessaires que je me contente d'inidiquer en passant : emploi de récipients préalablement stérilisés et maintenus pendant les observations à l'abri de toute contamination extérieure par les germes de l'air et surtout par ceux qui proviennent d'attouchements et de contacts non stériles. Tout cela présuppose, bien

(1) Je tiens à remercier tout particulièrement ici l'Institut de Laiterie de Kiel de l'impartialité qu'il a apportée dans l'étude de mon procédé, procédé qu'il a eu le soin de désigner par l'appellation : Alte-Ausführung (Vieux modèle). En effet, l'appareil qui a fonctionné à Kiel est un des deux premiers appareils construits au Danemark. Il présente plusieurs défauts de construction, lesquels ont été amendés par lo suite. 'Je me suis d'ailleurs expliqué sur ce point dans mon mémoire sur les appareils à plaques (Le Lait, février-mars 1931). 
entendu, la connaissance et la pratique parfaite de l'asepsie ; mais ce sont là données courantes pour ceux qui ont l'habitude du laboratoire de bactériologie.

Dans les laboratoires, en effet, tout le personnel, même le personnel subalterne, se soumet facilement, sous l'action des réflexes acquis par une bonne éducation, à tous les gestes qu'exigent de tels prélèvements. Mais pourquoi n'en serait-il pas de même dans l'industrie laitière, alors qu'il est si facile de disposer, partout où cela est nécessaire, de la vapeur exigée par la marche de cette industrie ? Il est rare que l'on voie dans les installations laitières, ici et là, aux endroits où elles pourraient être nécessaires, les prises de vapeur qui nous permettraient d'aseptiser sur place les récipients.

$$
\text { *** }
$$

Les différentes données à recueillir par la méthode comparative forment, selon moi, trois groupes bien distincts de critériums; chacun d'eux repose à son tour sur un certain nombre de facteurs qu'il faut nécessairement envisager et bien mesurer si l'on veut arriver à un jugement complet et impartial, capable de servir à une classification exacte dans l'examen des divers procédés de pasteurisation auxquels on peut être conduit. Voici les trois groupes de ces critériums :

a) Goût du lait.

b) Mesure du pourcentage de l'albumine coagulée.

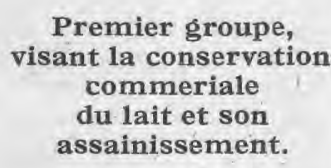

Deuxième groupe, relatif à

l'examen du lait lui-même. c) Durée de la conservation commerciale.

d) Dénombrement des microbes ; critique de certaines méthodes.

e) Mesure de l'a cidité du lait placé à l'étuve.

f) Recherche du B. coli.

g) Recherche du streptocoque hémolytique.

h) Contrôle de la disparition de la contagiosité d'un lait naturellement tubereuleux.

i) Déterminations physico-chimiques et biologiques : viscosité, $p H$, ferments solubles, etc.

j) Coagulation par la pré ure.

k) Séparation spontanée de la crème par le repos.

l) Conservation de l'acide carbonique ; analyse volumétrique de ce gaz ; détermination de l'acidité du lait avant et après chauffage.

m) Aération du lait.

n) Pouvoir bactéricide; pouvoir immunisateur.

o) Vitamines.

p) Recherches sur les produits de Ia vie de certains microbes aux températures de traitement du lait quand celui-ci est de longue durée. 


$$
\begin{array}{c|c}
\text { Troisième groupe, } \\
\text { relatif à }
\end{array} \quad\left\{\begin{array}{l}
\text { q) } \text { Conduite des appareils. } \\
\text { r) Consommation de vapeur et d'eau. } \\
\begin{array}{c}
\text { laspect économique } \\
\text { du traitement }
\end{array}
\end{array} \quad \begin{array}{l}
\text { s) Facilité du nettoyage. } \\
\text { t) Encombrement des appareils. }
\end{array}\right.
$$

\section{PREMIER GROUPE DE CRITERIUIMS RELATIFS A LA CONSERVATION COMMERCIALE DU LAIT ET A SON ASSAINISSEMIENT.}

A. Le goût du lait. - Il est certain qu'un lait pasteurisé, pour être reconnu bon à consommer, doit posséder un goût différant aussi peu que possible de celui d'un lait cru naturel recueilli dans de bonnes conditions.

Le procédé employé par la Station danoise d'Hilleröd dans l'examen du lait stassanisé (1) pour permettre au dégustateur de se prononcer sans être le moins du monde prévenu, est certainement très bon. Il eonsiste à donner à boire dans plusieurs verres en tous points semblables, sans la moindre marque, le lait pasteurisé à examiner et le lait cru qui lui correspond. Néanmoins, ce procédé peut donner lieu à quelques méprises, car l'acuité du sens du goût varie beaucoup d'un individu à un autre. Il est certainement très estompé chez les buveurs de vin et de boissons alcooliques en général, ainsi que chez ceux qui consomment beaucoup d'épices et chez les fumeurs ou priseurs de tabac. Aussi, peut-on estimer que l'épreuve destinée à fixer le goût du lait pasteurisé, par eomparaison avec le lait cru duquel il provient, doit se compléter d'épreuves plus précises, et e'est le cas de celles qui suivent.

\section{B. Détermination du pourcentage de l'albumine coagulée.}

- Il est nécessaire, évidemment, de connaître le taux de la même albumine dans le lait cru correspondant, afin de fixer ce pourcentage avec exactitude. Il y a lieu tout d'abord de choisir la méthode la plus sûre et en même temps la plus facile à employer pour arriver à des résultats comparables entre eux, parce que les chiffres obtenus avec une même matière première peuvent être assez différents.

Je puis fournir un exemple en ce qui concerne mes propres observations. D'après la méthode suivie à la Station danoise d'essais d'Hilleröd, le lait traité par le procédé de la couche mince n'aceuserait que $8 \%$ d'albumine coagulée, alors que d'après les déterminations faites sous ma direction à Strasbourg par M. Christen, le lait traité par le même procédé, aceuserait un pourcentage de $20 \%$ d'albumine coagulée. Ce procédé, qui est celui que l'on enseigne dans les écoles françaises de fromagerie, me paraît cependant digne d'être choisi, à condition toutefois que l'on opère

(1) 8 e rapport officiel. Trad. française dans Le Lait, 1930, 10, 382, 493 et 728. 
sur un volume moins exigu de lait. D'ailleurs, dans l'examen comparatif que M. Christen avait fait des différents procédés de pasteurisation, en suivant pour eux, bien entendu, la même méthode de dosage de l'albumine coagulée, cet auteur a trouvé, en face du taux de $20 \%$ pour le procédé de la couche mince, un taux de $36 \%$ pour le lait ayant été soumis à la pasteurisation basse et, enfin, un taux de $80 \%$ pour celui qui avait subi la pasteurisation haute. Ces chiffres sont tout à l'avantage du procédé en couche minee.

\section{Voici la méthode en question :}

A $10 \mathrm{~cm}^{3}$ de lait, on ajoute $90 \mathrm{~cm}^{3}$ d'eau à $40^{\circ}$, plus $1 \mathrm{~cm}^{3} 5$ d'acide acétique à $10 \%$, en agitant vigoureusement. Après 5 minutes de repos, on filtre sur papier et on lave trois fois le précipité aveo de l'eau distillée froide.

Le filtrat est neutralisé exactement aveo de la soude vis-à-vis de la phtaléine du phénol, et lorsqu'on est arrivé à ce point, sans attendre, on ajoute $0 \mathrm{~cm}^{3} 3$ d'acide acétique à $10 \%$; puis on porte à chaud au bain-marie pendant 20 minutes environ, Un précipité apparaît, lequel est recueilli sur un filtre de papier et lavé ensuite plusieurs fois avec de l'eau distillée froide. Lorsque l'opération est terminée, filtre et précipité sont placés dans un petit ballon de KJELDAHL; on y ajoute $10 \mathrm{~cm}^{3}$ d'acide sulfurique concentré, de densité 1,84 , plus un cristal de sulfate de cuivre et quelques grains de pierre ponce. On chauffe sur un bec de gaz jusqu'à clarification eomplète, ce qui demande environ 3 à 4 heures.

Le liquide est complété jusqu'à former $50 \mathrm{~cm}^{3}$ dans un ballon jaugé ; on prend $10 \mathrm{~cm}^{3}$ de cette liqueur, on y ajoute de la soude et on distille sur $10 \mathrm{~cm}^{3} \mathrm{de} \mathrm{HCl}$ N/70 coloré au rouge de méthyle.

Après 20 à 30 minutes de distillation, on porte la liqueur chlorhydrique N/70 à l'ébullition et on titre l'excès d'acide par $\mathrm{NaOH} \mathrm{N} / 70$. Soit $n$ la quantité de centimètres cubes de soude employés. La différence $10-n$ équivaut au volume de $\mathrm{HCl} \mathrm{N} / 70$ neutralisé par l'ammoniaque libéré lors de la distillation après addition de soude, des $10 \mathrm{~cm}^{3}$ empruntés au contenu du Kjeldahl, complété à $50 \mathrm{~cm}^{3}$.

Chaque centimètre cube de HClN/70 correspond à 0 gr., 0002 d'azote. Pour calculer la quantité d'albumine renfermée dans $100 \mathrm{~cm}^{3}$ de lait, nous ferons, par conséquent, l'opération suivante : $(10-n) \times 0,0002 \times 5 \times 10 \times 6,25=a$ grammes d'albumine pour cent de lait $(6,25$ est le facteur de multiplication à employer pour passer de l'azote trouvé à l'albumine correspondante (1)).

Je conseille de tenir compte, dans cette détermination, des justes remarques faites par Morr (The Analyst, 1931, 56, 2 \&े 9).

C. Durée de la conservation commerciale. - Il semble tout naturel que cette durée puisse être appréciée par les moyens les plus simples dont on dispose en laiterie. Toutefois, afin de pouvoir l'établir, sur une base solide, propre à servir dans toutes les comparaisons que nous devons faire des différents procédés de pasteurisation en usage, il ne faut pas se servir des récipients usuels. Les

(1) C'est le facteur 6,25 et non le facteur 6,38, ce dernier étant celui que l'on utilise pour la easéine, matière protéique phosphorée, alors que l'albumine n'a pas de phosphore dans sa molécule. (Note de la rédaction.) 
bouteilles les plus scrupuleusement lavées dans les différents bains et rincées dans l'eau la plus pure dont une laiterie puisse disposer, les bidons nettoyés avec le plus de soins par les appareils les plus modernes, n'offrent pas une garantie absolue. L'emploi de récipients vraiment stériles, dont le remplissage doit être effectué avec toutes les règles de l'asepsie, s'impose. L'à peu près est à éliminer même pour cette détermination, détermination qui semble si simple : la durée commerciale de conservation du lait.

D. Dénombrement des germes dans le lait:̣̂pasteurisé. On a recours, le plus généralement, à cette détermination pour établir ce qu'on appelle le coefficient d'efficacité d'un procédé de pasteurisation. Ayant eu l'oceasion au cours d'une existence déjà longue, d'apprécier ce que vaut ce dénombrement tel qu'il est effectué le plus généralement, j'ai été conduit à m'en passer. C'est en physiologiste que je me suis efforcé de l'apprécier et, finalement, de l'éliminer. On ne trouvera pas dans mes travaux sur le lait la moindre indication se rapportant à un pourcentage quelconque de germes tués au cours de la pasteurisation. En voici les raisons:

Ma longue expérience personnelle m'a montré les difficultés qu'il y a à arriver aux mêmes chiffres si, à plusieurs reprises, sur le même échantillon, on procède aux mêmes opérations de dénombrement: Cependant, j'ai travaillé dans des laboratoires très riches de matériel, me permettant de disposer de pipettes très uniformes, et pour chaque dilution, d'une nouvelle pipette. J'ai toujours eu soin, également, de faire les dilutions successives avec du bouillon stérile, et non avec de l'eau distillée, comme cela est prescrit dans plusieurs administrations de certains pays, notamment la Hollande, afin d'éviter une action meurtrière possible de l'eau distillée à l'égard de quelques germes. Je me suis efforeé enfin de distribuer toujours le même volume de milieu nutritif, sous la même épaisseur, en ayant soin de placer les couches de milieu nutritif dans une atmosphère de même degré d'humidité. Malgré toutes ces précautions, je ne suis jamais arrivé à des résultats identiques pour le même lait, et je crois que je suis d'accord avec de nombreux bactériologistes de la laiterie. Beaucoup d'auteurs reconnaissent que des opérateurs soigneux et attentifs obtiennent avec le même lait des chiffres très différents avec la même manière de faire; il est nécessaire d'ajouter que dans bien des eas, on rencontre des espèces microbiennes qui forment chainettes et que l'agitation des échantillons peut désagréger plus ou moins à chaque dilution.

Aussi, dès les débuts de mes recherches sur la pasteurisation, je me suis aperçu de tout ce qu'avait de conventionnel, - je dirai même plus, d'arbitraire, - ce genre de déterminations micro- 
biennes. Si l'on trouve sur une plaque $x$ colonies, peut-on penser que ce chiffre ait une valeur probante? Si le milieu nutritif avait été autre, son $p \mathbf{H}$, différent, si les colonies se trouvaient plus près de la surface ou plus près du fond de la eouche nutritive, différents pourraient être les chiffres fournis par le dénombrement. A quel moment, ajouterai-je, doit-on arrêter le dénombrement pour que celui-ci réponde à une réalité? Dans ma note à l'Académie des Sciences du 8 décembre 1924, j'ai montré l'inanité de ces déterminations, précisément en suivant l'éclosion des colonies sur les plaques de gélatine lactosée, convenablement entretenues jusqu'au sixième jour et même plus tard encore.

Après tout ce qui vient d'être dit, on s'explique la fragilité de certaines conclusions qui veulent prétendre établir des différences dans la valeur des divers modes de pasteurisation, en s'appuyant sur des différences, parfois faibles, dans le dénombrement microbien.

E. Mlesure de l'acidité du lait placé à l'étuve. - La flore bactérienne qui subsiste dans un lait rationnellement pasteurisé étant essentiellement constituée par les ferments lactiques, je conseille, au lieu de perdre son temps à les dénombrer avec la certitude de n'arriver qu'à des résultats incomplets, de mesurer le degré d'acidité dû à la culture de ees mêmes ferments. Nous avons ainsi, et très aisément, des déterminations que nous pouvons dire exactes et parfaitement comparables.

Le lait à mettre à l'étuve est recueilli aseptiquement dans une série de tubes à essais stérilisés au four à flamber. Au moment de la prise d'échantillons, on flambe les cotons qui les bouchent, et quand les tubes ont reçu le lait dans lequel on veut suivre le développement de l'acidité, on remet les cotons en ayant la précaution de les maintenir un peu plus sortis, de sorte que, lorsqu'on utilisera le lait pour en déterminer l'acidité, on pourra retirer ces cotons à la main, sane l'aide d'une pince. Les tubes à essais sont placés à l'étuve, soit à $30^{\circ}$, soit à $37^{\circ}$; mais il est bien entendu qu'on devra indiquer la température à laquelle on aura recours, en vue des comparaisons à effectuer. On note l'acidité qui se développe, et qui suit une courbe facile à établir sur la base des accroissements de cette acidité en fonction du temps. Les déterminations se feront de trois heures en trois heures.

Cette épreuve est, à mon sens, la meilleure pour distinguer un lait de pasteurisation basse qui nous donne toujours la réaction de la peroxydase de Sтовсн positive, d'un lait cru. Avec le lait cru, l'augmentation sera rapide ; l'acidité pourra atteindre $30^{\circ}$ Soxhlet, soit environ $67^{\circ}$ Dornic, alors qu'avec le lait pasteurisé elle ne dépassera pas $1^{\circ} \mathrm{S}$., soit $2{ }^{\circ} 25 \mathrm{D}$.

Dans mes études sur le procédé de la couche mince comparé à ceux de la pasteurisation haute et de la pasteurisation basse, il m'a été facile d'avoir une exacte notion du peu de valeur qu'ont, par eux-mêmes, les dénombrements microbiens. 
De larges plaques de BoĚz en gélose lactosée étaient ensemencées, chacune avec $1 \mathrm{~mm}^{3}$ de lait eru, traité à $95^{\circ}$ dans un pasteurisateur danois (pasteurisation haute), à $63^{\circ}$ pendant 30 minutes (pasteurisation basse) ou, enfin, à $75^{\circ}$ pendant 12 à 14 secondes par le procédé de la couche mince.

Sur la plaque du lait cru, les colonies sont innombrables, et au bout de 20 heures, elles commencent même la digestion de la gélose. Sur la plaque de la pasteurisation haute, - pasteurisation très haute, puisqu'elle atteint $95^{\circ},-$ très peu de colonies, mais presque toutes sont très développées; cependant, le lait pasteurisé correspondant accusait au bout de 18 heures d'étuve une acidité lactique de $30^{\circ} 2 \mathrm{~S}$. ( $68^{\circ} \mathrm{D}$.), presque autant, dirai-je, que l'échantillon de lait cru dont l'acidité était de $39^{\circ} \mathrm{S}$. $\left(87^{\circ} \mathrm{D}\right.$.). Les chiffres correspondants pour la pasteurisation basse et pour le procédé de la couche mince étaient respectivement de $15^{\circ} 2 \mathrm{~S}$. $\left(34^{\circ} \mathrm{D}\right.$.) et $10^{\circ} 2 \mathrm{~S}$. ( $23^{\circ} \mathrm{D}$.). Or, au simple examen à l'oeil nu ou à la loupe des différentes plaques, on constate, sans la moindre hésitation, qu'à l'accroissement de l'acidité la plus faible dans le lait de pasteurisation en couche mince, soit $10^{\circ} 2 \mathrm{~S}$., correspond un ensemencement qui a donné une plaque plus riche en colonies que la plaque ensemencée avec du lait de pasteurisation basse. A la vérité, si les colonies sont plus nombreuses, elles sont beaucoup plus petites que sur la plaque du lait de pasteurisation basse. Pour plus de détails, je conseille de lire ma note à l'Académie des Sciences du 8 août 1924 ("Du mode d'action de la chaleur sur les ferments lactiques dans la pasteurisation du lait "), et mon mémoire déjà rappelé, où l'on trouve les photographies des plaques de Boêz dont il a été question. Je tiens également à insérer à cette place les résultats de comparaisons effectuées en 1929, d'après la méthode commune des dénombrements microbiens, par M. Christen. Ils offriront une idée de ce que peut donner cette méthode, même en y recourant avec le plus de soins possibles.

TABLEAU I.

EXAMENS DES ENSEMENCEMENTS PRATIQUéS AVEG LE LAIT DE LA LAITERIE CENTRALE DE $X$.

Ensemencement en gélatine (boîtes de PÉTRI).

Pasteurisation basse

Pasteurisation haute

Dénombrement après 3 jours et demi.

25.000 colonies par centimètre eube de 1.000 .000 de colonies par centimètre lait. cube de lait.

Dénombrement après 4 jours et demi.

47.000 golonies par centimètre cube.

1.070.000 colonies par centimètre cube. 
Remarques. - Les colonies du lait de pasteurisation haute sont plus prếcoces et atteignent des dimensions plus grandes.

Les colonies du lait de pasteurisation basse apparaissent moins précocement et atteignent des d,éveloppements plus réduits que les colonies de pasteurisation haute.

Dénombrement 7 jours après l'ensemencement.

Grand développement : environ 100.000 Le nombre de colonies n'a pas augmenté colonies par centimètre cube de lait. après l'examen précédent, datant de 4 jours, mais leur diamètre a dépassé, pour quelques-unes, plugieurs centimètres.

Les ensemencements en gélose lactosée dans les boîtes de Bợz, à $37^{\circ}$, donnent moins de colonies qu'en boîtes de Pétri. Le développement de ces colonies est très rapide, notamment avec le lait de pasteurisation haute, et il arrive parfois à produire déjà des digestions du milieu, avant 36 heures, ainsi que cela se passe souvent avec les colonies du lait cru. Cela nous montre que dans le procédé de la pasteurisation haute, si le nombre de germes qui disparaissent lorsque le chauffage atteint et dépasse $85^{\circ}$ est considérable, il y a, par contre, des microbes qui, par leur précoce et rapide développement, témoignent d'avoir échappé, tout à fait peut-on dire, à l'action du chauffage.

Comment mesurer l'acidité lactique? - Le procédé employé couramment en France est celui qui a été préconisé par DoRnIC. Comme j'ai travaillé en France, il était tout naturel que j'y eusse recours également; mais, dans notre cas, il s'agissait d'apporter au dosage titrimétrique de l'acide lactique une très grande précision, indispensable ici, et c'est pour cette raison que j'ai exprimé l'acidité trouvée en degrés Soxhlet.

Par le procédé Dornic, on opère sur un trop petit volume de lait : $10 \mathrm{~cm}^{3}$, et, autre inconvénient me semble-t-il, la dilution de la soude est trop forte, ce qui nuit non seulement à la netteté du virage, mais est, par soi-même, une cause d'erreur, car, ainsi que le fait remarquer SomMerFend dans la critique du procédé Thörner et Pfeiffer, où le lait est dilué avant le titrage, pour obtenir, pensent-ils, une plus grande sensibilité du virage, cette dilution a pour but de réduire l'acidité originelle du lait. SoLDNER, par dilution du lait, a obtenu, en effet, les titrages suivants :

$$
\begin{aligned}
& 100 \mathrm{~cm}^{3} \text { de lait } \\
& 100 \mathrm{~cm}^{3} \text { de lait }+100 \mathrm{~cm}^{3} \text { d'eau } 5,0 \mathrm{NaOH} 1 / 4 \text { Norm. } 1 / 4 \text { Norm. } \\
& 100 \mathrm{~cm}^{3} \text { de lait }+200 \mathrm{~cm}^{3} \text { d'eau } 4,4 \mathrm{NaOH} 1 / 4 \text { Norm. } \\
& 100 \mathrm{~cm}^{3} \text { de lait }+500 \mathrm{~cm}^{3} \text { d'eau } 3,7-\mathrm{NaOH} 1 / 4 \text { Norm. } \\
& 100 \mathrm{~cm}^{3} \text { de lait }+1.000 \mathrm{~cm}^{3} \text { d'eau } 3,5 \mathrm{NaOH} 1 / 4 \text { Norm. }
\end{aligned}
$$

SOLDNER démontre encore d'une manière très simple que la dilution conduít à une diminution d'acidité du lait, en ajoutant de l'eau à un lait qui, venant d'être titré par la soude en présence de phtaléine du phénol, a une coloration rose ; l'addition de l'eau fait virer le rose vers un rouge plus vif. 
Dès le début de mes recherches, j'ai donc adopté le procédé Soxhlet, lequel offre pour nous les deux avantages suivants : $1^{\circ}$ d'effectuer le titrage sur $50 \mathrm{~cm}^{3}$ au lieu de 10 seulement; $2^{\circ}$ de s'adresser à une solution alcaline beaucoup plus forte, ce qui conduit à une moindre dilution du lait.

Ces développements incidents étant terminés, je reviens à la détermination de l'acidité dans le lait pasteurisé. On sait combien je me suis appliqué, dans toutes mes recherches, à appeler l'attention sur la baisse plus ou moins sensible de l'acidité du lait déterminée par le chauffage de ce dernier, baisse dont l'importance varie avec la méthode de chauffage employée et les appareils dont on s'est servi. La chute d'acidité est, en grande partie, déterminée par le départ de l'acide carbonique. Or, cette chute, non seulement est tout à fait importante si l'on opère l'extraction totale de l'acide carbonique par le vide, mais elle est considérablement plus forte encore, comme j'ai eu à plusieurs reprises l'occasion de le constater, si cette extraction par le vide est effectuée sur des laits fermentés. Voici un tableau dont les résultats sont très suggestifs :

TABLEAU II.

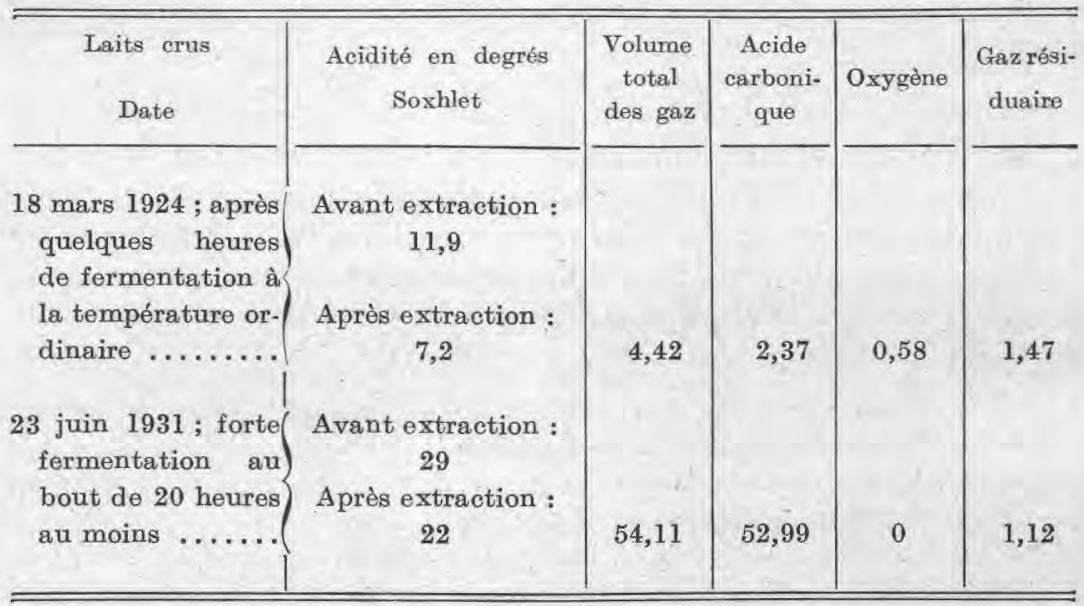

J'ai obtenu les mêmes résultats que ceux qui viennent d'être exprimés avec trois échantillons de lait provenant d'Italie et qui au bout de plus d'un mois, avaient une acidité plus forte encore, respectivement de $54^{\circ} 5,61^{\circ} 5$ et $83^{\circ} \mathrm{S}$. Dans tous ces cas, on n'a pas le droit de rapporter ces degrés si élevés d'acidité à l'acide lactique seul, ou ce serait tomber dans une grossière erreur. Au cours de la fermentation lactique, il se fait en même temps beaucoup d'acide carbonique, lequel intervient évidemment dans le dosage de l'acidité. 
F. Recherche du B. coli, - Le B. coli se trouve dans tous les laits crus. Son quantum est certainement beaucoup plus restreint dans les laits traits avec beaucoup de propreté, mais, quels que soient les soins d'asepsie que l'on apporte et quoi qu'on fasse, on en rencontre toujours. Quand on affirme le contraire, c'est qu'on n'a pas su le chercher. Le $B$. coli se trouve bien entendu en grande quantité dans le lait cru commun.

La présence du $B$. coli peut généralement ne pas représenter un grand danger pour l'hygiène, et la signification de cet organisme n'est peut-être pas la même que celle de la présence du même germe dans l'eau. Mais il importe cependant de dire que la disparition du $B$. coli dans un lait pasteurisé signifie, à défaut d'autre témoignage, que le traitement thermique a été d'une certaine efficacité à l'égard de l'assainissement du lait.

En fromagerie, la raréfaction du $B$. coli dans le lait pasteurisé présente déjà un grand intérêt, d'autant plus qu'à côté du $B$. coli et toujours avec lui, se trouve le $B$. aerogenes, lequel est responsable, plus peut-être que le $B$. coli, des incidents de gonflement que l'on constate dans les fromages en général, faits en partant de lait cru fortement pollué.

Dans le contrôle du lait alimentaire, la présence du $B$. coli est certainement la preuve d'une insuffisante pasteurisation. Le bacille de la fièvre typhoïde disparaît cependant dans ces conditions, mais d'une façon qui n'est pas absolument certaine, car le $B$. coli et les paracoli ne sont qu'un peu plus résistants à la chaleur que le bacille d'EBerth.

La disparition du $B$. coli est donc un point de repère très utile, un bon indice que d'autres germes plus ou moins pathogènes accusant à peu près le même degré de résistance, comme les Brucella, le B. diphtérique, le virus de la fièvre aphteuse, ont été également détruits. Par elle-même déjà, la disparition du $B$. coli est une garantie d'assainissement, ce microbe pouvant être la eause propre de certaines affections. Si l'on se donnait la peine de pratiquer l'hémoculture dans tous les cas, si fréquents, de ces fièvres d'origine alimentaire mises au compte d'aliments altérés, chez les enfants, peut-être trouverait-on souvent le $B$. coli évoluant sournoisement dans les vaisseaux, et notamment sur l'endocarde.

Les méthodes à suivre pour déceler la présence du $B$. coli dans le lait sont nombreuses; après en avoir pratiqué un certain nombre, je me suis arrêté à la plus simple et à la plus certaine, si l'on envisage très largement, sous son jour vrai, le but de la recherche.

Je pratique un très abondant ensemencement, - soit 10 gouttes de lait pour un petit tube de bouillon ou $10 \mathrm{~cm}^{3}$ de lait pour un petit matras du même bouillon. Si l'on se borne à l'ensemencement de quelques anses de platine, on risque avec 
un lait renfermant du $B$. coli, de n'en point trouver du tout. Point n'est besoin de milieu particulier, comme pour la recherche du $B$. coli dans les eaux (milieu phéniqué et bilié) ; le bouillon ordinaire suffit. Il est en effet un mauvais milieu de culture pour les ferments lactiques, mais il est très favorable au développement du B. coli; ce qui permet de retrouver celui-ci en pleine voie de multiplication, du deuxième au troisième jour. Rien n'est plus facile que de le reconnaître, d'abord par l'examen microscopique et ensuite par l'absence de coloration par le gram. Les amas de coccus constatés, qui ne prennent pas le gram, peuvent très bien ne pas toujours être du B. coli ; mais ce sont des espèces voisines : des paracoli ou du $B$. aerogenes, indésirables autant pour la fromagerie que pour la consomm on du lait pasteurisé.

Un lait entièrement. débarrassé de ses coli et paracolt, le serait certainement du bacille d'EBERTH, des Brucella, et du virus aphteux ; mais il peut garder intacte, s'il l'a possédée, sa contagiosité au regard du bacille de la tuberculose.

En suivant la technique très simple que je propose et en faisant l'examen au troisième jour après le séjour à l'étuve, on arrive très bien à isoler la culture de coccus ne prenant pas le gram, faite des espèces dont je viens de parler : soit le $B$. coli lui-même, soit un paracoli quelconque. Quoi qu'il en soit, dans un cas comme dans l'autre, la présence de ces microbes est l'indice d'une insuffisante pasteurisation.

Par la technique dont je viens de parler, il m'est arrivé souvent d'isoler dès le premier ensemencement sur plaque de gélose, le $B$. coli avec tous ses caractères bien connus. Mais si l'on n'y parvient pas tout de suite, voici les cas qui peuvent se présenter :

$1^{\circ}$ C'est le $B$. lactis aerogenes qui se développe toujours dans le lait envahi par les ferments lactiques. Cela complique la recherche du $B$. coli, bacille immobile ne prenant pas le gram et donnant une fermentation en bouillon sucré, avec formation de glycol butylénique et d'acétylméthylcarbinol. Pas d'indol en milieu peptoné non sucré.

$2^{\circ} \mathrm{Il}$ arrive souvent que $1^{\prime}$ 'on rencontre d'autres microbes appartenant au groupe Cloacae, ce qui complique aussi la recherche On a des colonies très grosses, d'un blanc jaunâtre et qui sur gélose sucrée, paraissent mucilagineuses et coulantes. Ce sont de petits bacilles très mobiles prenant le gram et donnant en bouillon sucré, une fermentation avec formation de glycol butylénique.

Un moyen qu'il faut considérer comme infaillible pour distinguer le $B$. coli, le lactis aerogenes et les microbes physiologiquement analogues, consiste à déterminer la nature de la fermentation què l'on observe, en bouillon sucré ou simplement en lait.

On prend soin d'agiter deux ou trois fois par jour les milieux en fermentation, pour en faciliter l'oxydation. On distille au $1 / \mathrm{s}$ le milieu mis à l'étuve pendant 3 à 5 jours à $30^{\circ}-35^{\circ}$ et sur le distillat, on recherche la réaction de l'jodoforme : 
A $5 \mathrm{~cm}^{3}$ de distillat, on ajoute une paillette d'iode représentant $0 \mathrm{gr} .1$ à $0 \mathrm{gr} .2$, puis 5 gouttes de lessive de soude.

S'il se forme rapidement, presque instantanément dirai-je, un précipité laiteux et abondant d'iodoforme, on se trouve en présence du $B$. lactis aerogenes, la réaction étant due à l'acétylméthylcarbinol que son métabolisme a formé.

Si le précipité n'est pas instantané, maís se forme très lentement, on se trouve en présence de $B$. coli, les corps générateurs d'iod oforme ne se formant que par une oxydation progressive.

\section{G. Recherche du streptocoque mastitidis et des streptoco-} ques hémolytiques. - Jusqu'à ces derniers temps, personne, peut-on dire, n'a attaché d'importance à la survie presque constante du streptocoque mastitidis dans les laits de pasteurisation basse, laits que l'on considérait comme les mieux assainis parmi les différents types de laits pasteurisés. On a pu tirer de ce fait la conclusion que ce streptocoque est un peu plus résistant au chauffage que le $B$. coli, lequel, par eontre, ne se trouve qu'exceptionnellement dans le lait de pasteurisation basse si celle-ci a été réalisée par de bons appareils et en suivant exactement les indications de la méthode. Cependant, la survie de ce streptocoque, très facile à confondre avee le $S$. thermophilus, ne devait pas tarder à inquiéter ceux des hygiénistes qui s'oceupent de pasteurisation du lait et dont le devoir était de signaler le danger que représente la présence de ce streptocoque dans le lait. Il n'est pas seulement l'hôte habituel de toute mastite, - accident si répandu dans l'espèce bovine, - mais il peut être facilement confondu avec des espèces beaucoup plus dangereuses que lui, tels que les streptocoques hémolytiques : $S$. puerperalis, $S$. erysipelatis et le $S$. epidemicus de l'angine infectieuse, microbe des plus sournois par son évolution intra-vasculaire, lente et insoupçonnée.

Ces streptocoques ne sont pas toujours faciles à identifier; e'est pourquoi, devant la grande fréquence de la mastite, nous estimons que l'hygiéniste conscient de son rôle est placé dans l'obligation de déconseiller la consommation du lait cru, quelle qu'en soit la provenance.

Dans une étable moderne, les vaches peuvent être sûrement indemnes de tuberculose, mais on n'a pas les mêmes assurances en ce qui concerne la mastite, aussi bien celle qui serait due à une variété streptococcique non hémolytique que celle dont l'évolution dépend d'une variété hémolytique. La mastite peut passer - et passe en effet - inaperçue pendant fort longtemps, malgré la vigilance la plus scrupuleuse de celui qui trait. Par conséquent, dans les premiers temps de son évolution; il n'est pas possible d'isoler la bête et de séparer son lait. Il peut s'agir d'une de ees mastites banales, qui seraient peut-être plus fréquentes avec la 
traite mécanique, et ce serait donc très coûteux de prendre les mesures nécessitées par l'isolement, avant que le diagnostic n'ait signalé la certitude du danger.

Le Lait a déjà eu la bonne idée, à plusieurs reprises, d'exposer les techniques les plus couramment employées pour cette délicate recherche, ce qui me dispense de m'étendre davantage sur ce point, cependant fort intéressant.

H. Contrôle de la disparition de la contagiosité dans un lait naturellement tuberculeux. - Pour pouvoir se prononcer sur l'efficacité d'un procédé de pasteurisation vis-à-vis de son complet assainissement, il faut, inévitablement, soumettre un lait naturellement tuberculeux à l'épreuve du procédé en examen. Il ne faut pas songer à l'emploi d'un lait quelconque dans lequel on répandrait des bacilles tuberculeux provenant d'une culture. Cette méthode est à écarter; d'un côté, parce que si le voile que les bacilles tuberculeux forment à la surface de leur culture n'est pas bien brisé, émietté, il peut y avoir de forts amas de bacilles échappant forcément à l'action du chauffage, ce qui fausserait les résultats; d'un autre côté, ces bacilles tuberculeux, lorsqu'ils proviennent d'une culture, ne sont pas entourés de cette coque glaireuse, de ces résidus cellulaires qui, en les protégeant, les soustraient facilement à l'action de la chaleur.

Nous ferons également remarquer qu'au cours des essais effectués, il faut éviter que les cobayes-témoins, e'est-à-dire ceux qui reçoivent le lait tuberculeux cru, ne meurent avant que les lésions tuberculeuses aient eu le temps de se développer, du fait d'infections secondaires provenant de la flore commune du dit lait. II importe done de détruire dans le lait cru les quelques variétés de microbes capables de provoquer de pareils accidents. On y arrive aisément, soit par un chauffage préalable modéré, qui n'atteint ni ne modifie sensiblement les bacilles tuberculeux, soit par l'emploi d'un antiseptique, ce qui demande une très grande connaissance du comportement de cet antiseptique en pareille circonstance.

Le Professeur O. Bang, de l'Ecole supérieure de Médecine vétérinaire et d'Agriculture de Copenhague, qui a exécuté quatre séries de contrôle avec du lait naturellement tuberculeux et pasteurisé par le procédé de la couche mince, à la Station officielle d'essais d'Hilleröd (Danemark), évite ces infections secondaires en mélangeant, soit au lait tuberculeux, soit aux «boues " provenant de la centrifugation de ce même lait, un composé (l'antiformine) à base d'hypochlorite de soude.

DEUXIÈME GROUPE DE CRITERIUMS RELATIFS A L'EXAMEN DU LAIT.

I. Données physico-chimiques et biologiques. - Je me 
borne à conseiller les deux seules déterminations qui, dans mes essais comparatifs ayant porté sur le lait de pasteurisation en couche mince, de pasteurisation basse et de pasteurisation haute, vis-à-vis, bien entendu, - ai-je besoin de le dire, - du même lait cru de mélange, m'ont donné des résultats probants : la détermination de la viscosité et celle du $p$ H.

Les variations que l'on enregistre du eôté des autres données physico-chimiques, comme la conductivité, l'abaissement cryoseopique, ne sont ni nettes ni mesurables. Je n'ai constaté nul abaissement du point cryoscopique dans aucun des trois types de lait pasteurisé étudié. Je dois cependant noter, sans insister, qu'après un séjour à $-2^{\circ}$, alors que les échantillons - de lait cru étaient congelés au bout de 1 ou 2 jours, les échantillons de lait pasteurisé en provenance de ce lait cru, dans les mêmes conditions de temps et de température, se maintenaient liquides.

Viscosité. - Les viscosités par rapport à l'eau sont déterminées au viscosimètre d'OSTWALD. J'ai constaté une légère augmentation de la viscosité, proportionnelle au degré de durée du chauffage subi par le lait. C'est ainsi que la viscosité, qui dans le lait cru est 1,69 , devient 1,72 dans le lait pasteurisé en couche mince, 1,74 dans le lait de pasteurisation basse et 1,76 dans le lait de pasteurisation haute.

La détermination $d u p H$. - Le chauffage, quel qu'il soit, augmente l'acidité ionique du lait; par conséquent, plus un lait a été modifié par le chauffage, plus le $p H$ est bas et s'éloigne forcément de la valeur qu'il a dans le lait cru d'origine. Cette détermination de l'abaissement du $p H$ n'est guère réalisable à l'aide des indicateurs colorés. Les différences qui portent sur la seconde décimale ne peuvent être appréciables que par la méthode potentiométrique, laquelle est délicate et exige un appareillage assez coûteux.

Les ferments solubles. - Dans le lait cru, il y a peroxydase et réductase, affectées, aussi bien l'une que l'autre, par le chauffage et détruites par celui-ci même quand il est suffisant. C'est la recherche de la peroxydase qu'il convient de choisir comme point de repère et d'adopter comme test du degré de conservation du lait après le chauffage. La raison de ce choix est facile à comprendre : la peroxydase a son origine au sein de la mamelle, en même temps que tous les autres principes qui entrent dans la composition du lait, alors que la réductase est produite par les microbes qui cultivent dans le lait.

Jusqu'ici, la constatation de la peroxydase par la réaction de SтоRCH a servi pour distinguer d'emblée un lait oru d'un lait pasteurisé, parce que, le plus généralement, la pasteurisation 
pratiquée étant la pasteurisation haute, la peroxydase était détruite par elle.

Mais l'entrée en scène des nouveaux procédés de pasteurisation n'a pas été sans étonner les techniciens de ce que j'appellerai la vieille laiterie, parce qu'ils ne trouvaient plus dans la réaction de STORCH le test qui leur permettait de distinguer le lait cru du lait pasteurisé. La pasteurisation haute détruisant la peroxydase, la pasteurisation basse y touchant moins, et moins encore le proc dé de la couche mince, on ne peut pas faire de la réaction de STоRсн le critérium de l'assainissement du lait. Je prendrai même le contre-pied de l'opinion ancienne, en disant que meilleure sera la conservation de la réaction de SтоRсн dans son intensité initiale, alors que l'assainissement du lait est assuré par une bonne destruction microbienne, meilleure en même temps sera la conservation de tous les équilibres du lait et des autres principes biochimiques qu'il renferme. Si l'on veut s'assurer qu'un lait est vraiment pasteurisé, il n'y a qu'à le soumettre à l'épreuve de l'étuve. L'acidité du lait cru monte rapidement, ainsi que je l'ai indiqué plus haut; celle du lait pasteurisé, - à condition, bien entendu, qu'il n'ait pas été pollué ultérieurement, - monte au contraire lentement, surtout pendant les trois premières heures.

J'ajouterai que pour un homme de laboratoire, il est d'autres moyens d'investigation.

J. Caillage du lait par addition de présure. - Ce critérium a une double portée : l'une de caractère pratique, l'autre de caractère plutôt biologique. Il intéresse au plus haut point les fromageries, car, depuis quelque temps, la pasteurisation du lait tend à se répandre dans cette industrie. Naturellement, on ne pouvait songer à recourir au lait de pasteurisation haute, parce qu'il est trop modifié sous l'action de la chaleur; il ne pouvait done être utilisé pour la fabrication des fromages, l'emprésurage conduisant à des résultats déplorables. Il est vrai qu'on peut, dans une certaine mesure, remédier à ce grave inconvénient, par l'addition au lait avant l'emprésurage, de doses variables de chlorure de calcium. Cela est devenu une pratique assez courante dans la fromagerie.

Il est certain que l'épreuve d'emprésurage peut servir de critérium au fromager avisé, pour lui permettre de faire son choix parmi les procédés de pasteurisation susceptibles d'être utilisés dans son industrie.

Dans l'étude très générale que j'ai faite en 1910 au sujet de l'action du chauffage sur le lait vis-à-vis des modifications que ce chauffage peut apporter dans l'aptitude du lait à cailler par la présure, je pus saisir ce fait singulier, qui a ensuite été reconnu 
exact par plusieurs expérimentateurs, à savoir que l'action du chauffage préalable du lait sur le temps que ce dernier met ensuite à coaguler par la présure, n'est pas toujours retardatrice. Il existe un optimum de température et de durée de chauffage qui facilite l'action labique au lieu de l'amoindrir. En d'autres termes, le lait caille plus vite que l'échantillon correspondant de lait cru. Voici, pour être plus précis, le rappel des paragraphes où je consignais, en 1910 , cette constatation tout à fait imprévue au moment où j'écrivais :

("..... L'action d'une cuisson modérée (55 à $\left.65^{\circ}\right)$, au lieu de retarder la coagulation du lait, l'accélère nettement. Du lait qui caille en 50 minutes se prend en masse en 20 minutes seulement, toutes choses égales d'ailleurs : quantité de lait, température, réaction, etc., lorsqu'il a été chauffé pendant 15 minutes à $55^{\circ}$ ou $65^{\circ}$. Avec le chauffage à $70^{\circ}$, le lait se montre sensible à l'action de la présure dans la même mesure que le lait cru. Ensuite, au delà de $70^{\circ}$, l'action empêchante apparaît et augmente parallèlement à l'action de la température. »

J'ai tenu à préciser ce fait en reproduisant textuellement les termes par lesquels je le faisais connaître, parce que ce fait, en apparence insignifiant, se rattache à celui de la séparation spontanée de la crème dans le lait au repos.

K. Séparation spontanée de la crème dans le lait au repos. - En effet, l'ascension de la crème est également retardée par le chauffage qu'a subi le lait, et dans un lait trop chauffé, l'écrémage spontané est extrêmement restreint; il manque en effet - ou presque - dans les laits de pasteurisation haute, et même dans les laits de pasteurisation basse, pour peu que l'on dépasse la température de $63^{\circ}$ ou qu'on maintienne celle-ci plus de temps qu'il n'est indiqué.

Or, nous observons pour la séparation spontanée de la crème du lait au repos, le même phénomène que celui qui vient d'être signalé au sujet de la coagulation par la présure ; par un chauffage convenable, l'ascension de la matière grasse se fait mieux et plus vite que dans le lait cru et la ligne de crème est nettement marquée. Cette notion de l'optimum, que nous relevons pour le caillage du lait par la présure et pour la séparation spontanée de la crème du lait laissé au repos, nous la retrouvons dans nombre de réactions biologiques; elle est classique dans l'étude des diastases. D'ailleurs, quelque hypothèse que l'on puisse formuler sur l'accélération du caillage du lait par la présure sous l'action de la chaleur, ainsi que sur l'accélération, pour ainsi dire parallèle, que l'on note dans l'écrémage du même lait laissé au repos, ces deux effets d'un chauffage modéré nous paraissent être les témoignages les plus certains du bon état 
de conservation de la structure du lait, tout au moins de celle de certains de ses composants qui peuvent nous apparaître parmi les plus fragiles vis-à-vis du chauffage.

Si une bonne séparation spontanée de la crème dans un lait pasteurisé a, commercialement, un très grand avantage, parce qu'elle prouve à la ménagère que le lait qui lui est vendu n'a pas, préalablement, été plus ou moins écrémé, elle correspond, en même temps, ̀̀ une parfaite conservation du lait. En d'autres termes, l'état particulier où se trouvent les constituants protéiques les plus fragiles du lait, pour donner une séparation spontanée normale de la crème, est la preuve la plus frappante que le chauffage n'a pas été brutal.

Cette séparation spontanée normale de la crème est certainement la démonstration indiscutable d'une bonne pasteurisation, telle qu'on est arrivé à la réaliser avec les meilleurs appareils de la moderne pasteurisation basse. On va même plus loin en Angleterre et, surtout, aux Etats-Unis, puisqu'une bonne montée de la crème est une condition indispensable dans l'acceptation d'un nouveau procédé de pasteurisation.

Mais j'exprimerai l'idée qu'il ne suffit pas qu'il y ait seulement formation d'une bonne couche de crème dans un lait pasteurisé, pour avancer que la pasteurisation a été effectuée rationnellement. Pour que cette formation soit effectivement le témoignage, l'expression d'une bonne pasteurisation, e'est-à-dire pour qu'elle signifie que le lait en cause a subi le traitement le plus rationnel, le plus voisin de l'idéal, il faut que la séparation de la crème se produise également plus précocement que dans le lait cru correspondant, laittémoin, placé dans les mêmes conditions que le lait pasteurisé.

Je ne sache pas qu'aucun appareil de pasteurisation, avant mon premier appareil industriel, toujours conservé comme appareil"type " à la Laiterie centrale de Strasbourg, nous ait donné un lait qui présente ce phénomène d'une séparation plutôt précoce de la crème. Ce fait, conditionné par un chauffage préalable à une température déterminée, a été remarqué, en outre, - pas avant moi peut-être, puisque je l'ai signalé dès 1924, mais sûrement indépendamment de moi, - par W. REID. Dans un travail relativement récent de cet auteur (Milk Dealer, 1927), analysé au Bulletin mensuel des Renseignements frigorifiques de juillet-août 1928 et reproduit par Le Lait, sur l'effet de différentes températures de pasteurisation sur plusieurs des propriétés physiques du lait, je constate en effet que si $W$. ReID ne dit rien du phénomène proprement dit de la précocité de la montée de la crème, il signale sans le moindre doute, avec beaucoup de précision même, le phénomène qui accompagne cette précocité, à savoir la formation d'une couche de crème plus 
épaisse, dans un lait chauffé à une température convenable. En deça, et de très peu, comme d'ailleurs au delà de la température optima, l'épaisseur de la couche de crème diminue. Voici d'ailleurs le paragraphe que je crois utile d'emprunter à l'analyse reproduite par Le Lait, pour mieux fixer le côté, en quelque sorte historique, du phénomène si intéressant sur lequel je tiens à attirer l'attention des savants et des techniciens des questions laitières :

"La hauteur de la colonne de crème diminue avec chaque accroissement de la température de pasteurisation, sauf avec celle de $142^{\circ}$ F. $\left(61^{\circ}\right.$ C. $)$, qui fournit, en moyenne, $0,47 \%$ en volume de crème de plus que la température de $140^{\circ}$ (60 C.). ")

Il importe donc de se maintenir dans des limites étroites de température, de durée et de conditions de chauffage, qui caractérisent ce que nous pourrions appeler l'optimum d'action thermique. C'est pourquoi j'ai toujours très fortement insisté près du constructeur de mes appareils pour que ces derniers soient fabriqués dans des conditions qui permettent la réalisation de cet optimum.

Une belle épaisseur de la couche de crème et surtout, peut-être, la précocité dans la séparation de la crème du lait écrémé, voilà des faits intéressants qui peuvent être la traduction de ce qu'on peut appeler la pasteurisation idéale.

L. Analyse volumétrique de l'acide carbonique et des constituants de l'air dans le lait pasteurisé. - Dans le lait qui résulte d'une élaboration d'un liquide spécial aux dépens des éléments apportés par le sang, on retrouve, quoique en proportions moindres, l'acide carbonique du sang circulant. Sur la fonction chimique de l'acide carbonique dans le lait, je me suis déjà expliqué plus haut. A mon avis, un lait appaurri plus ou moins en acide carbonique, - et cela dépend du procédé de pasteurisation auquel on l'a soumis, - possède une valeur alimentaire moindre, dont la diminution est mesurable, précisément en fonction de l'acide carbonigue retenu. Il est d'autant plus utile de déterminer quel est le volume de l'acide carbonique qui reste dans le lait, soit à l'état de gaz libre, soit à l'état combiné, - ce qui nous amène à faire une analyse volumétrique des constituants du lait, - que lors du chauffage de ce liquide par les procédés usuels, si le lait s'appauvrit d'un côté en acide carbonique, il s'enrichit de l'autre en oxygène, oxygène dont on n'ignore plus les inconvénients, non seulment à l'égard du goût, mais en ce qui concerne la conservation des vitamines.

Pour la technique à suivre, on peut se reporter à ma note à l'Académie des Sciences sur ce sujet. Je me réserve de publier ultérieurement, avec beaucoup de détails, un travail d'ensemble sur les gaz du lait. 
M. Aération du lait. - J'ai déjà signalé, au début de cet article, les méfaits de l'aération du lait sur ses vitamines; il n'est donc pas nécessaire que je revienne sur ce point. C'est aux hygiénistes, à ceux qui doivent juger de la valeur d'un procédé de pasteurisation, de tenir compte de la remarque que j'ai présentée, et qui me paraît digne de retenir l'attention.

N. Présence et état de conservation du pouvoir bactéricide. - Au cours de ces dernières années et dans de nombreux travaux, il a été beaucoup question du pouvoir bactéricide du lait cru fraîchement trait; on peut donc dire que c'est une chose que tout le monde connait, ou, du moins, dont tout le monde parle. Il convient toutefois de faire remarquer que la preuve expérimentale de ce pouvoir n'a pas encore été fournie d'une façon indéniable. On parle d'une phase bactéricide, phase qui serait caractérisée par le fait que le nombre des bactéries dont le lait se trouve chargé à sa sortie même de la mamelle, diminue pendant quelques heures, au lieu d'augmenter. Cette diminution s'accentue dans le lait gardé à basse température, mais elle finit par passer inaperęue dans le lait porté à l'étuve dès sa sortie de la glande.

Dans cette phase intervient certainement, en tout premier lieu, le phénomène de l'adaptation, phénomène dont les bactériologistes sont à même d'apprécier la valeur. Les coccus mammaires, en réalité, changent de milieu, en abandonnant le parenchyme glandulaire ; de plus, pour des êtres de l'ordre des microbes, ne pas se multiplier c'est, assez souvent, mourir. Mais il y a encore d'autres facteurs dont j'ai eu l'occasion de m'occuper au cours de ces dernières années. L'un d'eux est le principe lytique de l'espèce bactériophage, dont il est très difficile de suivre le développement, développement qui s'arrête par le fait de l'acidification du milieu.

Le facteur essentiel, très caractéristique de la phase bactéricide, est sans doute un anticorps qui se trouve dans le lait, s'y comportant à la manière de tous les anticorps microbiens, d'une façon élective sur les coccus mammaires et, en plus faible proportion, sur le $B$. coli. C'est ce que j'ai pu mettre en évidence par addition d'une alexine fraîche (sérum non chauffé de cobaye) à des laits pasteurisés ou vieillis. Cette addition déclenchait alors la manifestation du pouvoir bactéricide en question, disparu dans le lait ci-dessus, par suite du chauffage ou du vieillissement.

BoRDET avait déjà montré qu'on rencontre dans le lait ce même principe lytique pour les bactéries communes de l'air, principe que Flemming et Aluixon ont trouvé dans des humeurs de l'organisme : les larmes, le mucus nasal, ainsi que dans le blane d'œuf.

Par contre, le principe bactéricide, que j'ai pu mettre en évidence, a un caractère plus électif, au point de pouvoir jouer le rôle d'un 
véritable anticorps microbien. Comme tel, il peut intervenir à son tour pour contribuer à la défense de l'intestin du nourrisson envers les infections intestinales.

Quoi qu'il en soit, constater l'existence de ce principe dans un lait pasteurisé est d'un réel intérêt, car c'est au moins apporter la preuve que le lait a été très modérément modifié par le chauffage qu'il a subi. Et, à ce titre, cela mérite de figurer parmi les critériums les plus importants pour la classification des différents procédés de pasteurisation.

Ainsi que je l'ai déjà montré, il est facile de mettre en évidence la survie de ce principe dans le lait pasteurisé en couche mince, alors que sa disparition est certaine dans les laits pasteurisés usuels.

o. Vitamines. - La détermination de l'état de conservation des vitamines dans un lait pasteurisé est de celle qu'il n'est pas donné à tout le monde de mener à bien; il faut une très grande pratique de ce genre de recherches pour arriver à des résultats probants, car avec les petits animaux de laboratoire, on se heurte à la très grande difficulté de leur faire ingérer des quantités assez importantes de lait, lesquelles seraient nécessaires pour qu'on puisse juger de la marche de la nutrition.

A priori, il est admissible que le lait de vache ne contienne que peu de vitamines par centimètre eube; aussi, pour commencer à noter une influence quelque peu favorable sur le cobaye en état de carence, par l'ingestion de lait de vache cru, il faut au moins que l'animal arrive à en ingérer $80 \mathrm{~cm}^{3}$, ce qu'il n'est pas toujours facile de réaliser. On remédie un peu à cette difficulté inhérente à l'emploi des petits animaux pour ce genre d'études, en introduisant dans leur alimentation des régimes mixtes. Pour remédier, par exemple, à l'insuffisance du lait, même cru, en vitamine $C$, on ajoute du jus d'orange ; pareillement, pour remédier à l'insuffisance de ce même lait en vitamine antibéribérique, on ajoute encore de la levure de bière. Il en résulte une très grande eomplication, amenant beaucoup d'erreurs, en général involontaires, de la part du personnel ; il faut s'occuper soi-même de l'alimentation de ses animaux de laboratoire.

Dans l'étude préliminaire que je faisais il y a plus de dix ans pour me rendre compte de l'état de conservation des vitamines dans du lait de vache stérilisé par le procédé de la couche mince, à $140^{\circ}$, e’est ainsi que je procédai, me chargeant moi-même de l'alimentation des petits, cobayes. J'obtins des résultats favorables, et, en effet, malgré la très haute température à laquelle le lait avait été porté, les vitamines $A$ et $B$ restèrent presque intactes ; pour la vitamine $C$, les résultats que j'obtins étaient contradictoires.

Ces constatations, plus que satisfaisantes, en raison de la très 
forte épreuve subie par le lait, puisqu'il s'agissait de le stéritiser, m'ont dispensé de les reprendre avec le lait simplement chauffé à $75^{\circ}$, en vue de le pasteuriser. J'ajouterai que l'absence d'aération dans le procédé de la couche mince était un autre garant de la faible altération que devaient subir les vitamines lors de la pasteurisation par ce procédé. En effet, avec tous les autres procédés de pasteurisation, y compris ceux à plaques ou par chauffage en tubes où l'on estime pouvoir faire également de la couche mince, le lait est plus ou moins aéré, ce qui ne peut que nuire aux vitamines qu'il contient.

- Au surplus, les expériences du Professeur Frédericra, de Copenhague, directeur de l'Institut d'Hygiène de l'Université, pour les vitamines A et B, et celles du Docteur M. V. Poulsen, chef de service à l'Hôpital des Enfants, pour la vitamine C, ont établi la plus parfaite conservation de ces trois vitamines dans le lait traité par mon procédé de pasteurisation. Le lait stassanisé était fourni au Professeur Frederiora et au Professeur Poulsen par la Station officielle d'essais d'Hilleröd.

P. Recherches sur les produits de la vie de certains microbes (souches de subtilis et coccus lactiques thermophiles acidoprotéolytiques). - J'ai déjà eu l'occasion de mentionner le cas de ces différentes espèces de microbes que l'on trouve, en abondance parfois, dans le lait cru, et qui cultivent à des températures relativement hautes - celles auxquelles a recours la pasteurisation basse. Les produits de la vie de ces microbes qui se multiplient à leur aise dans le lait, pendant le traitement thermique qu'on lui fait subir dans le but de l'assainir, doivent forcément se retrouver dans le lait de pasteurisation basse, particulièrement au sortir de certains dispositifs. Dans quelle mesure ces produits peuvent-ils être contraires à l'hygiène du consommateur ? Il serait intéressant de les rechercher, de les déceler, et même, si on le pouvait, de les titrer.

\section{TROISIEMME GROUPE DE CRITÉRIUMS RELATIFS A L'ASPECT ECONOMIQUE DU TRAITEMENT THERMIQUE.}

Q. Conduite des appareils. - La conduite des appareils est un point des plus intéressants pour le prix de revient du lait pasteurisé, ainsi que pour la constance des résultats à obtenir dans l'assainissement du lait. Avec la pasteurisation haute et avec la pasteurisation basse, la vigilance doit être de tous les instants. Si, comme on le fait souvent dans l'appareillage de pasteurisation basse, cette vigilance est confiée à l'automatisme, comme c'est le cas pour l'ouverture et la fermeture des vannes des chambreurs, on s'expose à des défaillances dont les conséquences peuvent être plus ou moins sérieuses.

R. Consommation de vapeur et d'eau. - Ces deux points 
de vue, qui peuvent paraître secondaires, n'influent, en réalité, que sur le prix de revient du lait, et non pas sur l'assainissement de ce liquide, assainissement qui est le but essentiel de la pasteurisation. Cependant, si on peut arriver aux mêmes résultats avec des dépenses moindres, le gain réalisé à la fin de l'année peut être important.

S. Facilité du nettoyage. - C'est une question intéressant autant le côté économique que le but de la pasteurisation lui-même, puisque avec un appareil d'un nettoyage difficile, on peut toujours s'attendre à des résultats regrettables.

T. Encombrement des appareils et possibilités de leur disposition. - Les appareils de pasteurisation basse qui exigent des bâtiments à plusieurs étages, des dispositifs en cascades, dispositifs que les Américains ont beaucoup eontribué à répandre, doivent être écartés ; les frais de premier établissement deviennent en effet, avec eux, considérables. Par contre, les systèmes peu encombrants, qui n'exigent pas plusieurs étages, qui donnent du lait sortant sous pression du circuit qu'il vient de traverser, ce qui peut permettre de le distribuer soit au même étage, soit même un peu plus haut que les appareils de pasteurisation eux-mêmes, offrent certainement de grands avantages économiques. Le prix de construction de l'immeuble destiné à la laiterie sera ainsi réduit dans des proportions considérables.

U. IPrix de revient. - Le prix de revient doit être mis en face des facteurs suivants :

a) Les avantages du nettoyage ;

b) La facilité de conduite des appareils ;

c) La consommation en eau et en vapeur ;

d) Le coût de l'immeuble.

\section{REMARQUES ET CONGLUSIONS}

Tous les critériums que je viens d'examiner doivent être envisagés par qui veut, en toute conscience et pleine connaissance de cause, se faire une opinion exacte sur la valeur d'une méthode, d'un appareil, d'un procédé de pasteurisation du lait.

Il en est parmi eux qui ne peuvent pas être aussi facilement étudiés que les autres ; certains, en effet, ne peuvent être établis qu'à la suite de déterminations demandant l'emploi d'appareils délicats et un personnel spécialisé. Toutefois, si nous procédons à un examen attentif de tous ces critériums, nous nous apercevons que quelquesuns d'entre eux peuvent être considérés comme étant la conséquence d'un ou de plusieurs autres plus faciles à définir, à soumettre à l'analyse, comme l'est, par exemple, la mesure de l'acidité du lait et de ses variations. De la détermination de cette mesure, dépend étroitement l'étude de la conservation de l'acide carbonique, à 
laquelle se rattache la question de l'aération du lait, qui en est vraiment la conséquence.

$\mathrm{Si}$ nous parlons de maintenir l'acidité du lait, ce qui sousentend la conservation de son acide carbonique originel, nous sommes amené à envisager parallèlement le critérium de la conservation des vitamines, puisque par la conservation de l'acide carbonique, on évite la fâcheuse action combinée du chauffage et de l'aération, dont j'ai parlé plus haut.

Aussi, de tous les critériums ci-dessus, et en laissant de côté ceux qui sont d'ordre économique, je ferai un choix de 11, dont la détermination me paraît essentielle pour qu'on puisse se prononcer d'une façon complète et impartiale sur la valeur d'un procédé de pasteurisation, voire d'un simple pasteurisateur :

$1^{\circ}$ Détermination du goût : pas de goût de cuit.

$2^{\circ}$ Détermination du pourcentage d'albumine soluble coagulée : faible proportion.

$3^{\circ}$ Bonne durée de conservation commerciale du lait, en récipients stériles.

$4^{\circ}$ Marche de l'acidification du lait à l'étuve : très lente jusqu'à 3 à 5 heures.

$5^{\circ}$ Disparition du B. coli.

$6^{\circ}$ Disparition du $B$. mastitidis et de tout streptocoque hémolytique.

$7^{\circ}$ Contrôle de l'assainissement d'un lait naturellement tuberculeux.

$8^{\circ}$ Conservation du degré d'acidité initiale du lait cru, après la pasteurisation.

$9^{\circ}$ Bonne conservation de l'aptitude du lait à cailler par la présure.

$10^{\circ}$ Conservation de l'aptitude à une bonne séparation spontanée de la crème dans le lait au repos, et, de plus, constatation d'une certaine précocité de cette séparation, cette précocité s'accompagnant d'une épaisseur un peu plus grande de la couche de crème.

$11^{\circ}$ Conservation de la peroxydase : réaction positive de Storch.

L'établissement de ces critériums exige une parfaite entente entre savants et praticiens s'intéressant à la laiterie. J'exprime le souhait que cette entente conduise à l'étude des méthodes de recherches que l'on devra suivre pour être admis à porter un jugement sérieux sur la valeur des appareils de pasteurisation donnant le plus de garanties à l'égard de l'hygiène et le plus d'avantages de toutes sortes à l'égard de la pratique industrielle. 